\title{
Aortic thrombus in a patient with myeloproliferative thrombocytosis, successfully treated by pharmaceutical therapy: a case report
}

\author{
Hidesuke Yamamoto ${ }^{1,2^{*}}$, Haruaki Nishimaki ${ }^{1}$, Norikazu Imai ${ }^{1,2}$, Masakazu Nitta ${ }^{2}$, Osami Daimaru ${ }^{1}$
}

\begin{abstract}
Introduction: Thrombosis in myeloproliferative thrombocytosis occurs usually in the microvessels and mediumsized arteries and veins and only rarely in the aorta. Aortic thrombosis is usually treated with thrombectomy. Reported here is a rare case that was treated pharmacologically.

Case presentation: A 60-year-old Japanese woman presented with numbness of both lower extremities. Her platelet count was $1787 \times 10^{3} / \mu \mathrm{l}$. Through bone marrow examination, we diagnosed her condition as myelodysplastic and/or myeloproliferative disorder-unclassifiable. Abdominal ultrasonography and computed tomographic scan revealed aortic thrombosis. Her platelet count was controlled with hydroxyurea and ranimustine. Aspirin and ticlopidine improved the numbness in both lower limbs on the second day. Aortic thrombosis was not observed in a computed tomographic scan on the seventh day.
\end{abstract}

Conclusion: For aortic thrombosis, surgical management is usually adopted, but pharmacological management is also an option because of its immediate curative effects.

\section{Introduction}

Thrombocytosis is classified into myeloproliferative thrombocytosis and reactive thrombocytosis. In myeloproliferative thrombocytosis, there is always a risk of thrombosis. It is well known that thrombosis in myeloproliferative thrombocytosis occurs in the medium-sized arteries and veins and in the microvessels [1]. However, aortic thrombosis is rare [2,3]. We report a rare case of aortic thrombosis with myeloproliferative thrombocytosis. The main therapeutic method to resolve aortic thrombosis is a thrombectomy $[4,5]$. However, the aortic thrombus in our case was treated immediately pharmacologically. This case may serve as a reference for the choice of therapy for aortic thrombosis.

\section{Case presentation}

A 60-year-old Japanese woman presented with numbness in both lower extremities. This symptom appeared four months prior to admission, and she experienced giddiness two months before. However, she had ignored

\footnotetext{
* Correspondence: hide1005@aichi-med-u.ac.jp

'Department of Internal Medicine, Division of Hematology, Daiyukai General Hospital, 1-9-9 Sakura, Ichinomiya, Aichi 491-8551, Japan
}

these symptoms. Her medical history revealed that she had undergone an oophorectomy, and she had a uterine sarcoma at the age of 36 and mastitis at the age of 45 . Her consciousness was clear but she had anaemic conjunctivae. Physical examination revealed that she was a well-nourished female with normal vital signs. She had no chest pain. Her liver and spleen were not palpable. Although she experienced numbness in both her lower limbs, the neurological findings were normal, and neither extremity was cold.

The patient had a white-blood-cell count of $2.74 \times$ $10^{3} / \mu$ l with $37 \%$ neutrophils, $62 \%$ lymphocytes and $1 \%$ monocytes. Her haemoglobin level was $7.8 \mathrm{~g} / \mathrm{dl}$ and her platelet count was $1787 \times 10^{3} / \mu$ l. Examination of bonemarrow aspirate revealed normal cellular marrow, megakaryocytic hyperplasia and erythroid dysplasia. Her karyogram was normal.

Abdominal ultrasonography revealed in the aorta a moveable mass with a diameter of $1.5 \mathrm{~cm}$. The interior of the mass had no blood supply. A computed tomographic scan of her abdomen revealed a circular thrombus in the descending aorta at the level of the 


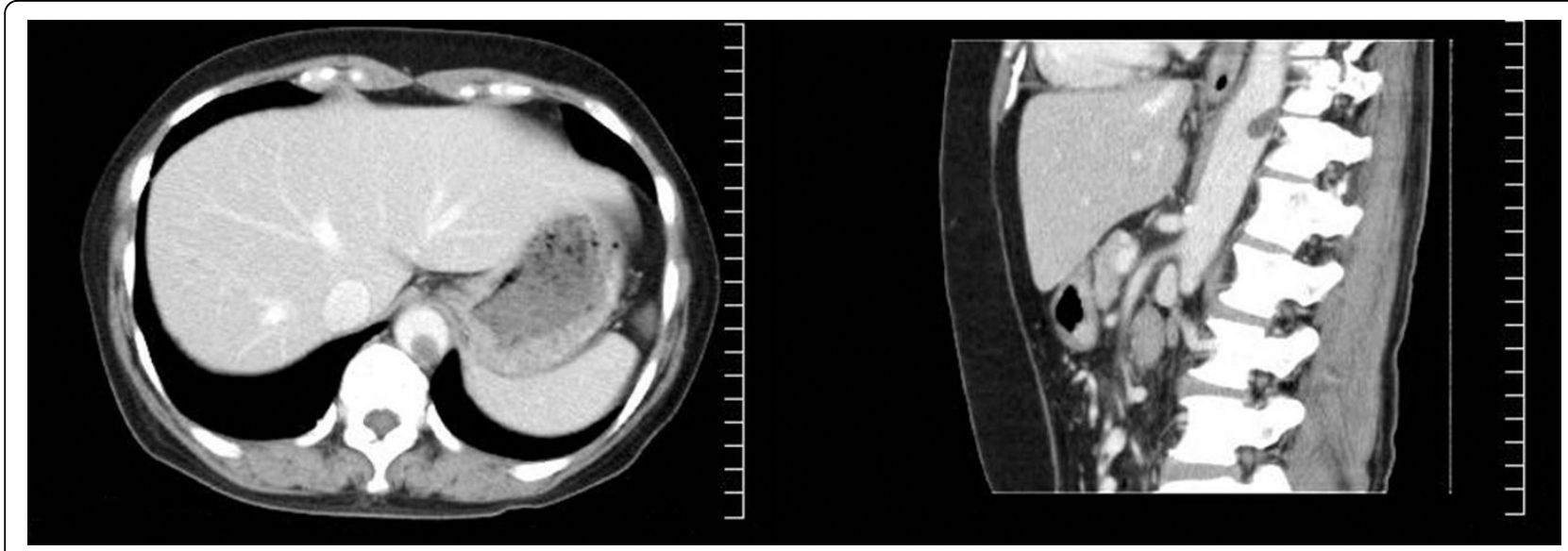

Figure 1 Enhanced computed tomography scan before treatment. A thrombus is observed in the lower-line aortic blood vessel at the level of the diaphragm.

diaphragm (Figure 1). Arteriosclerosis was not recognized at that point.

We diagnosed this case of thrombocytosis and anemia as myelodysplastic syndrome and/or myeloproliferative disorder-unclassifiable (MDS/MPD-U), and the aortic thrombus was thought to be associated with myeloproliferative thrombocytosis.

For myeloproliferative thrombocytosis, she was treated with $500 \mathrm{mg}$ of hydroxyurea from the seventh day of her hospital stay. However, she was administered 1000 mg of hydroxyurea on the $16^{\text {th }}$ day because her platelet count increased to $2074 \times 10^{3} / \mu \mathrm{l}$. In addition, she was administered $50 \mathrm{mg}$ of ranimustine on the $18^{\text {th }}$ day. The platelet count decreased to $274 \times 10^{3} / \mu \mathrm{l}$ on the $39^{\text {th }}$ day, but it increased slowly thereafter. After administration of $1000 \mathrm{mg}$ of hydroxyurea, her platelet count stabilized at approximately $1000 \times 10^{3} / \mu$ l. She was administered transfusions for anemia.

Ticlopidine was administered to prevent the development of a thrombosis on the seventh hospital day, which was the same day treatment for myeloproliferative thrombocytosis was initiated. Aspirin (100 mg) was administered when an aortic thrombus was identified by computed tomographic scan on the $10^{\text {th }}$ day of her hospital stay. The numbness in both of her lower extremities disappeared within two days. Also, the aortic thrombus was not observed in the computed tomographic scan on the $17^{\text {th }}$ day of her hospital stay. She was administered aspirin after seven days (Figure 2). She has been without symptoms for more than one year.

\section{Discussion}

MDS is haematological monoclonal disease characterized by dysplasia and ineffective haematopoiesis, which causes cytopenia [6]. Chronic myeloproliferative disorders (CMPD) are also haematological monoclonal diseases. However, they are characterized by an increase in the number of more than one type of myeloid lineage. There is a disease group that borders or overlaps both the MDS and CMPD disease groups. The World Health Organization (WHO) defined MDS/MPD in 2001 [7]. MDS/MPDs were classified as chronic myelomonocytic leukaemia, atypical chronic myeloid leukaemia, juvenile myelomonocytic leukaemia and myelodysplastic and/or myeloproliferative disordersunclassifiable (MPD/MDS-U). MDS/MPD-U fails to meet the criteria for any of the specific MPD/MDS entities. On one hand, it is characterized by dysplasia and ineffective haematopoiesis, and on the other hand, it is characterized by an increase in the number of more than one type of myeloid lineage. A representative disorder of this group of diseases is refractory anaemia with ringed sideroblasts (MDS-RARS), wherein the platelet count is over $600 \times 10^{3} / \mu \mathrm{l}[8]$. The symptoms in this case are similar to those in the case of MDS-RARS and it satisfies the criteria for MPD/MDS; however, this case cannot precisely be diagnosed as MDS-RARS because Prussian blue reaction was not performed. The new WHO classification was published in September 2008 [9]. As for MDS/MPD, the name was changed to myelodysplastic and/or myeloproliferative neoplasm (MDS/ MPN). According to the new classification, this case is a case of MDS/MPN-U.

It is well known that thrombosis occurs in mediumsized vessels and in microvessels in myeloproliferative thrombocytosis [1]. Of these thrombosis cases, including cases of cerebral infarction, transient cerebral ischaemic attack, retinal thrombosis, ischaemic heart disease, pulmonary infarction, hepatic artery and portal vein thrombosis, deep venous thrombosis and ischaemia of limbs, $11 \%$ to $25 \%$ are included in the myeloproliferative thrombocytosis group [10]. However, cases of aortic 


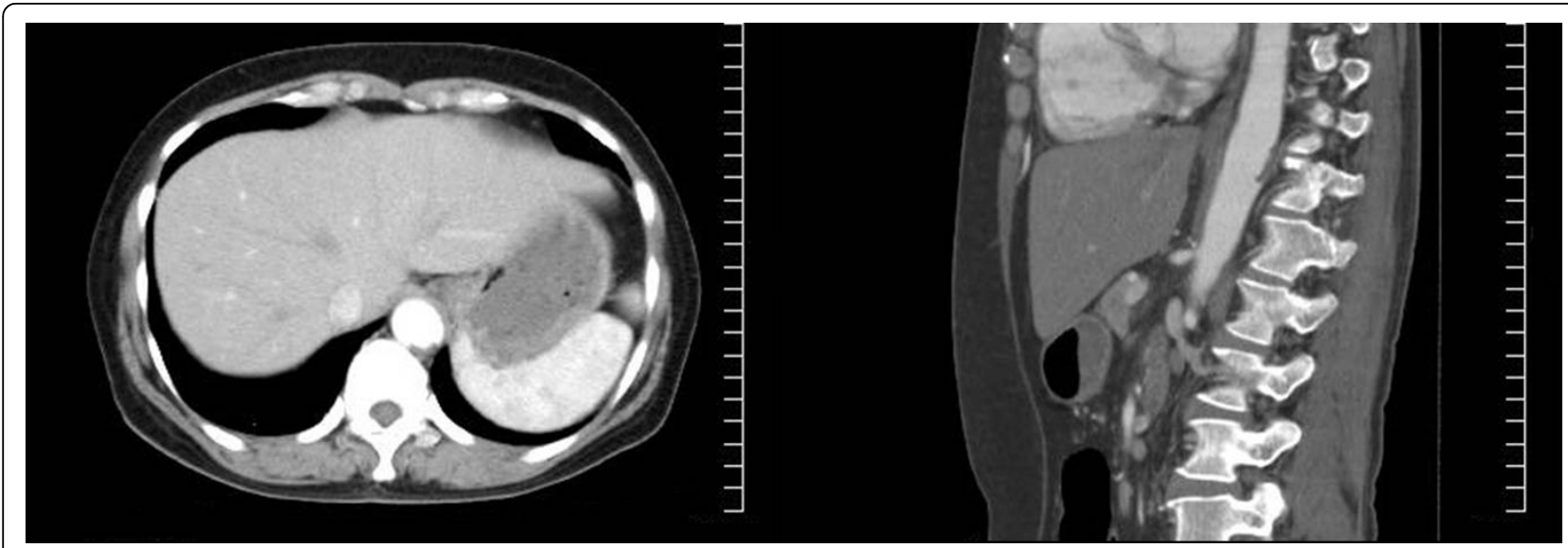

Figure 2 Enhanced computed tomography scan after seven days of treatment. The circular thrombus has disappeared. However, there is a low density area in the same spot, which was identified as a wall thrombosis.

thrombosis are very rare among the myeloproliferative thrombocytosis cases [2,3]. Therefore, the stipulated treatment has not been established.

The prognosis of patients with myeloproliferative thrombocytosis is mainly determined by the onset of thrombosis; therefore, it is very important to control thrombosis [11]. Usually, in a case of aortic thrombosis, surgical management is adopted as the main therapy, and therapy in the form of medication is rare.

Johnson et al reported an essential thrombocythaemiaassociated intra-aortic thrombus that was treated with an aortic thrombectomy, and they removed the thrombus, which consisted of platelets [4]. Sohn et al also described a case of surgical management [5]. This treatment is advantageous as surgical management prevents necrosis in the organ by an embolism in the peripheral arteries. However, aortic thrombectomy is a risky procedure. Ehrenfeld et al reported the case of a patient who died of shock after an aortic thrombectomy for thrombocytosis [12].

On the other hand, Fang et al reported a large aortic thrombus with essential thrombocythaemia that was treated with medication, and to the best of our knowledge, this is the only case in which this was done [13]. In this case, the symptoms disappeared within two days (similar to our case), and the disappearance of the thrombus was confirmed three weeks later by computed tomography. In our case, we confirmed the resolution of the thrombus eight days later. Because the symptoms disappeared within two days in both cases, the resolution of the thrombus might have occurred at similar times in both cases. If the thrombus can be dissolved early by pharmacological treatment, we believe that not only aortic thrombectomy but also pharmacological treatment can prevent necrosis in the organ. This implies that pharmacological treatment can be used as the primary treatment for this condition in the future.

Johnson et al identified a thrombus comprising platelets; this thrombus was termed a 'white clot' due to the characteristic thrombocythaemia [4]. Therefore, it is logical to treat patients with aspirin which is a cyclooxygenase (COX) inhibitor. Aspirin disrupts thromboxan A2 production by inhibiting COX-1 of the arachidonic acid cascade, and it exhibits antiplatelet action [14]. In particular, secondary aggregation, most of which result in the formation of large thrombi, such as an aortic thrombosis, is prevented. On the other hand, ticlopidine prevents platelet aggregation by specifically obstructing the adenosine diphosphate receptor P2Y12 [15]. In addition, it prevents shear-induced platelet aggregation. This case indicated that the combination of aspirin and ticlopidine can act synergistically because of their different mechanisms. In this case, there is a possibility that two types of antiplatelet agents contributed to early action.

This case showed that antiplatelet medications are effective for aortic thrombosis, and this case may serve as a reference in the choice of therapy for aortic thrombosis.

\section{Conclusion}

We report an aortic thrombosis case with myeloproliferative thrombocytosis treated by medical management. Thus far, surgical management has been adopted for such cases. However, this report suggests that medical management is a safe and effective strategy for aortic thrombosis in the case of myeloproliferative thrombocytosis.

\section{Consent}

Written informed consent was obtained from the patient for publication of this case report and any accompanying 
images. A copy of the written consent is available for review by the Editor-in-Chief of this journal.

\section{Abbreviations}

CMPD: chronic myeloproliferative disorder; COX: cyclooxygenase; MDS: myelodysplastic syndrome; MDS/MPD: myelodysplastic/myeloproliferative disorder; MDS/MPN: myelodysplastic/myeloproliferative neoplasm; RARS: refractory anaemia with ringed sideroblasts; U: unclassifiable; WHO: World Health Organization.

\section{Author details}

'Department of Internal Medicine, Division of Hematology, Daiyukai General Hospital, 1-9-9 Sakura, Ichinomiya, Aichi 491-8551, Japan. 'Department of Internal Medicine, Division of Hematology, Aichi Medical University School of Medicine, 21 Karimata, Yazako, Nagakute, Aichi 480-1195, Japan.

\section{Authors' contributions}

$\mathrm{MN}$ and $\mathrm{OD}$ analyzed and interpreted the patient data regarding the haematologic disease. $\mathrm{NI}$ and HN participated in the clinical evaluation of the patient. HY obtained written informed consent from the patient, carried out the literature search and drafted the manuscript. All the authors reviewed and approved the final manuscript.

\section{Competing interests}

The authors declare that they have no competing interests.

Received: 23 October 2009 Accepted: 21 July 2010

Published: 21 July 2010

\section{References}

1. Watson KV, Key N: Vascular complications of essential thrombocythaemia: a link to cardiovascular risk factors. Br J Haematol 1993, 83:198-203.

2. Mitus AJ, Schafer Al: Thrombocytosis and thrombocythemia. Hematol Oncol Clin North Am 1990, 4:157-178.

3. Valla D, Casadevall N, Lacombe C, Varet B, Goldwasser E, Franco D, Maillard JN, Pariente EA, Leporrier M, Rueff B, Muller O, Benhamou JP: Primary myeloproliferative disorder and hepatic vein thrombosis. A prospective study of erythroid colony formation in vitro in 20 patients with Budd-Chiari syndrome. Ann Intern Med 1985, 103:329-334.

4. Johnson M, Gernsheimer T, Johansen K: Essential thrombocytosis: underemphasized cause of large-vessel thrombosis. J Vasc Surg 1995, 22:443-449.

5. Sohn V, Arthurs Z, Andersen C, Starnes B: Aortic thrombus due to essential thrombocytosis: strategies for medical and surgical management. Ann Vasc Surg 2008, 22:676-680.

6. Brunning RD, Bennett JM, Frandrin $G$, et al: Myelodysplastic syndromes.

7. World Health Organization Classification of Tumors, et al: Pathology and Genetics, Tumor of Hematopoietic and Lymphoid Tissues Lyon: IARC PressJaffe WS 2001, 61-74.

8. Vardiman JW, Pierre R, Bain B, World Health Organization Classification of Tumors, et al: Myelodysplastic/myeloproliferative diseases. Pathology and Genetics, Tumor of Hematopoietic and Lymphoid Tissues Lyon: IARC PressJaffe WS 2001, 45-60.

9. Gupta R, Abdalla SH, Bain BJ: Thrombocytosis with sideroblastic erythropoiesis: a mixed myeloproliferative myelodysplastic syndrome. Leuk Lymphoma 1999, 34:615-619.

10. Swerdlow SH, Campo E, Harris NL: World Health Organization Classification of Tumours of Haematopoietic and Lymphoid Tissues. Lyon: IARC Press 2008.

11. Harrison CN: Platelets and thrombosis in myeloproliferative diseases. Hematology Am Soc Hematol Educ Program 2005, 409-415.

12. Cortelazzo S, Viero P, Finazzi G, D'Emilio A, Rodeghiero F, Barbui T: Incidence and risk factors for thrombotic complications in a historical cohort of 100 patients with essential thrombocythemia. J Clin Oncol 1990, 8:556-562

13. Ehrenfeld M, Penchas S, Eliakim M: Thrombocytosis in rheumatoid arthritis. Recurrent arterial thromboembolism and death. Ann Rheum Dis 1977, 36:579-581.
14. Fang M, Agha S, Lockridge L, Lee R, Cleary JP, Mazur EM: Medical management of a large aortic thrombus in a young woman with essential thrombocythemia. Mayo Clin Proc 2001, 76:427-431.

15. Goto S: Understanding the mechanism of platelet thrombus formation under blood flow conditions and the effect of new antiplatelet agents. Curr Vasc Pharmacol 2004, 2:23-32.

16. Gachet C: ADP receptors of platelets and their inhibition. Thromb Haemost 2001, 86:222-232.

doi:10.1186/1752-1947-4-219

Cite this article as: Yamamoto et al:: Aortic thrombus in a patient with myeloproliferative thrombocytosis, successfully treated by pharmaceutical therapy: a case report. Journal of Medical Case Reports $20104: 219$

\section{Submit your next manuscript to BioMed Central and take full advantage of:}

- Convenient online submission

- Thorough peer review

- No space constraints or color figure charges

- Immediate publication on acceptance

- Inclusion in PubMed, CAS, Scopus and Google Scholar

- Research which is freely available for redistribution 